\title{
Article \\ Identification of an Orally Bioavailable, Brain-Penetrant Compound with Selectivity for the Cannabinoid Type 2 Receptor
}

\author{
Meirambek Ospanov ${ }^{1,2}{ }^{\mathbb{D}}$, Suresh P. Sulochana ${ }^{3} \mathbb{D}$, Jason J. Paris ${ }^{3}{ }^{(D}$, John M. Rimoldi $^{3}$, Nicole Ashpole ${ }^{3}$, \\ Larry Walker ${ }^{1}$, Samir A. Ross ${ }^{1,3} \mathbb{D}$, Abbas G. Shilabin $4, * \mathbb{D}$ and Mohamed A. Ibrahim 1,*D
}

check for updates

Citation: Ospanov, M.; Sulochana, S.P.; Paris, J.J.; Rimoldi, J.M.; Ashpole, N.; Walker, L.; Ross, S.A.; Shilabin, A.G.; Ibrahim, M.A. Identification of an Orally Bioavailable, Brain-

Penetrant Compound with Selectivity for the Cannabinoid Type 2 Receptor. Molecules 2022, 27, 509. https:// doi.org/10.3390/molecules27020509

Academic Editor: Raffaele Capasso

Received: 21 December 2021

Accepted: 11 January 2022

Published: 14 January 2022

Publisher's Note: MDPI stays neutral with regard to jurisdictional claims in published maps and institutional affiliations.

Copyright: (C) 2022 by the authors. Licensee MDPI, Basel, Switzerland. This article is an open access article distributed under the terms and conditions of the Creative Commons Attribution (CC BY) license (https:/ / creativecommons.org/licenses/by/ $4.0 /)$.
1 National Center for Natural Products Research, School of Pharmacy, The University of Mississippi, University, MS 38677, USA; mospanov@olemiss.edu (M.O.); lwalker@olemiss.edu (L.W.); sross@olemiss.edu (S.A.R.)

2 Department of Chemistry and Technology of Organic Substances, Natural Compounds and Polymers, Al-Farabi Kazakh National University, al-Farabi Ave. 71, Almaty 050040, Kazakhstan

3 Department of BioMolecular Sciences, School of Pharmacy, University of Mississippi, University, MS 38677, USA; spsuloch@olemiss.edu (S.P.S.); parisj@olemiss.edu (J.J.P.); jrimoldi@olemiss.edu (J.M.R.); nmashpol@olemiss.edu (N.A.)

4 Department of Chemistry, East Tennessee State University, Johnson City, TN 37614, USA

* Correspondence: shilabin@etsu.edu (A.G.S.); mmibrahi@olemiss.edu (M.A.I.); Tel.: +423-439-6917 (A.G.S.); +662-915-1147 (M.A.I.); Fax: +423-438-5835 (A.G.S.); +662-915-1006 (M.A.I.)

\begin{abstract}
Modulation of the endocannabinoid system (ECS) is of great interest for its therapeutic relevance in several pathophysiological processes. The CB2 subtype is largely localized to immune effectors, including microglia within the central nervous system, where it promotes anti-inflammation. Recently, a rational drug design toward precise modulation of the CB2 active site revealed the novelty of Pyrrolo[2,1-c][1,4]benzodiazepines tricyclic chemotype with a high conformational similarity in comparison to the existing leads. These compounds are structurally unique, confirming their chemotype novelty. In our continuing search for new chemotypes as selective CB2 regulatory molecules, following SAR approaches, a total of 17 selected (S,E)-11-[2-(arylmethylene)hydrazono]-PBD analogs were synthesized and tested for their ability to bind to the CB1 and CB2 receptor orthosteric sites. A competitive $\left[{ }^{3} \mathrm{H}\right] \mathrm{CP}-55,940$ binding screen revealed five compounds that exhibited $>60 \%$ displacement at $10 \mu \mathrm{M}$ concentration. Further concentration-response analysis revealed two compounds, $4 \mathbf{k}$ and $\mathbf{4 q}$, as potent and selective CB2 ligands with sub-micromolar activities $\left(K_{\mathrm{i}}=146 \mathrm{nM}\right.$ and $137 \mathrm{nM}$, respectively). In order to support the potential efficacy and safety of the analogs, the oral and intravenous pharmacokinetic properties of compound $\mathbf{4 k}$ were sought. Compound $4 \mathbf{k}$ was orally bioavailable, reaching maximum brain concentrations of $602 \pm 162 \mathrm{ng} / \mathrm{g}$ (p.o.) with an elimination half-life of $22.9 \pm 3.73 \mathrm{~h}$. Whether administered via the oral or intravenous route, the elimination half-lives ranged between 9.3 and $16.7 \mathrm{~h}$ in the liver and kidneys. These compounds represent novel chemotypes, which can be further optimized for improved affinity and selectivity toward the CB2 receptor.
\end{abstract}

Keywords: cannabinoid receptors CB1/CB2; pyrrolobenzodiazepines; neuroinflammation; neurodegenerative diseases; central nervous system (CNS); pharmacokinetics (PK); radioligand binding assay

\section{Introduction}

Neurodegenerative disorders are a widespread cause of morbidity and mortality worldwide, characterized by the slow, progressive damage/loss of neurons in the central nervous system (CNS), which is associated with deficits in function (e.g., movement, memory, cognition) that are related to the affected CNS region(s) [1]. The progression of many neurodegenerative diseases is thought to be driven by the template-directed misfolding, seeded aggregation, and cell-cell transmission of characteristic disease-related proteins, 
leading to the consecutive spreading of pathological protein aggregates [2]. Modulating the activity of endocannabinoids (ECB) has held therapeutic promise for treating a wide range of diseases associated with inflammation and oxidative stress. The cannabinoid receptors subtype 2 (CB2), which were identified molecularly in 1993, have been the subject of considerable attention, primarily due to their promising anti-inflammatory potential without the adverse psychotropic effects more commonly associated with CB1 receptorbased therapies [3]. Many studies have further corroborated the in vivo link between chronic neuroinflammation and CB2 upregulation in animal models of pain [4] and inflammation [5]. These studies raise the possible logical connection between CB2 receptors and immunological function [6]. Activation of CB2 receptors by ligands favors a range of receptor conformations that can affect different signaling pathways [7].

Administration of the $\mathrm{CB} 2$ antagonist was able to decrease the neuroprotective effects of beta-caryophyllene (BCP) and strengthen the need for $\mathrm{CB} 2$ activation. The distribution of the CB2 subtype in neural cell types (activated astrocytes, reactive microglia, oligodendrocytes, perivascular microglia, and neural progenitor cells), many of which are integral to blood-brain barrier function, supports its potential role in CNS integrity [7]. CB2 agonists have shown promising neuroprotection using in vitro (BV-2 microglial cell lines and primary microglia cultures) and in vivo models (murine model) of neurodegeneration. The potential of CB2 agonists as neuroprotective agents has been particularly sought given the lack of psychotropic adverse effects generally seen with CB1 agonists [8]. One of the limitations of the small molecule modulators of CB2 has been issues with selectivity and differential activity depending on cell type/species. Several 'selective' CB2 agonists have been shown to regulate orphan cannabinoid receptors and/or opioid receptors or other off-target proteins. In addition, recent evidence suggests that inverse agonists of $\mathrm{CB} 2$ may also be neuroprotective. A synthetic CB2 inverse agonist protects neurons from excitotoxic insult in vitro and in vivo [9]. SMM-189 prevented the rat primary microglia-mediated inflammation and was neuroprotective against N-methyl-D-aspartic acid (NMDA)-induced neuronal excitotoxicity in brain-mimicking neuron-glia primary cultures obtained from rat hippocampal tissues [9]. Concurrent development of novel, selective CB2 inverse agonists, partial, and full agonists would allow for a closer comparison of the effects of these two mechanistically distinct pharmacological effects so the role of CB2 in neuroinfectious diseases can be elucidated.

Novel synthetic and natural compounds with neuroprotective potential have emerged as new pharmaceuticals in the management of many neurological conditions, presenting a heterogeneous chemical structure and varied pharmacological activity, which may be useful in the treatment of high-complexity diseases. The search for new drug leads that target endocannabinoid signaling is challenging as endocannabinoids are implicated in various physiological and pathological processes.

Pyrrolo[2,1-c][1,4]benzodiazepines (PBDs) are a class of natural products known to possess anti-tumor and antibiotic activities [10-12]. Molecular modeling studies have shown that the PBD tricyclic structure is well-suited to dock within the catalytic cavity of $\mathrm{CB}$ receptors, allowing them to interact with the endocannabinoid signaling system. As a part of our continuing efforts to identify novel neuroprotective agents through a structure-based rational design and multi-step synthetic approach [13], the present project focused on the synthesis of seventeen analogs of (S,E)-11-[2-(arylmethylene)hydrazono]PBD ligands (4a-4q) as potential CB2 selective regulators that may serve as novel and effective neuroprotectants (Figure 1). Discovery of novel regulatory compounds using structure-activity relationship (SAR) studies will advance the field of neuroprotection and neurorestoration by providing information necessary to determine if this class of compound is a viable chemotype for the design of potentially new therapies to treat neurodegenerative disorders in humans. 


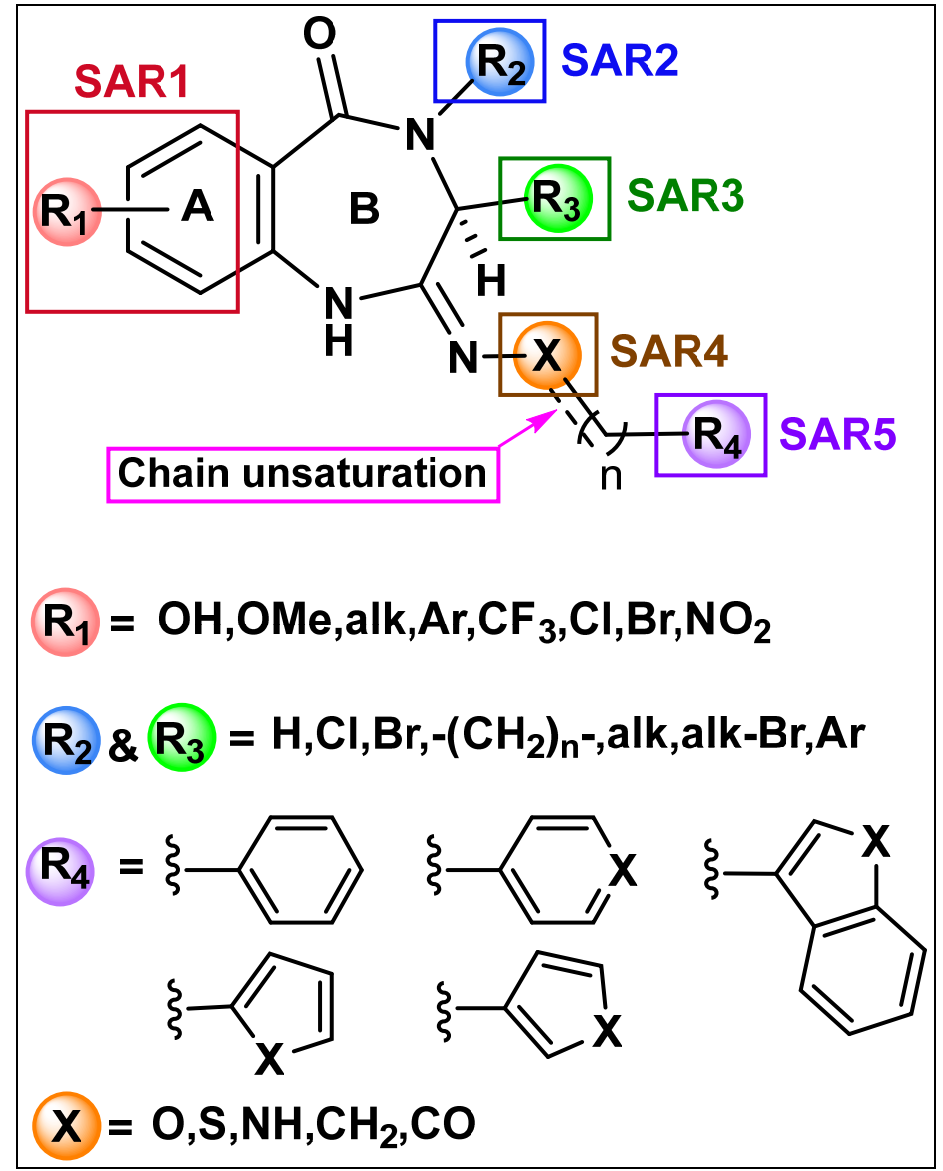

Figure 1. Structures of the proposed analogs.

Drug discovery programs routinely perform pharmacokinetic (PK) studies in mice to prioritize lead compounds based on anticipated exposure-efficacy and exposure-toxicity relationships. Promising lead compounds with selective binding affinities were examined for their pharmacokinetics properties in plasma or tissue (brain, liver, or kidney) following oral or intravenous administration.

\section{Material and Methods}

General Experimental Procedures

The ${ }^{1} \mathrm{H}$ and ${ }^{13} \mathrm{C}$ NMR spectra were recorded in DMSO- $d_{6}$ and $\mathrm{CDCl}_{3}$ on Bruker 400 and $500 \mathrm{MHz}$ spectrometer operating at 400, $500 \mathrm{MHz}$ for ${ }^{1} \mathrm{H}$ and $100,150 \mathrm{MHz}$ for ${ }^{13} \mathrm{C}$ NMR. Chemical shift $(\delta)$ values are presented in ppm and in reference to the residual solvent signals of DMSO- $d_{6}$ and $\mathrm{CDCl}_{3}$ at $\delta_{\mathrm{H}} / \delta_{\mathrm{C}} 2.50 / 39.5$ and 7.25/70.2, respectively. The coupling constants value $(\mathrm{J})$ reported in $\mathrm{Hz}$.

LC analysis was conducted using an Agilent 1100 HPLC system, RP-C18 column $\left(150 \times 4.6 \mathrm{~mm}\right.$; particle size $5 \mu \mathrm{m}$; Luna) with column oven temperature set at $25^{\circ} \mathrm{C}$ and a gradient system of eluent water (A) and acetonitrile (B). The gradient condition was as follows: $0-2 \mathrm{~min}(5 \% \mathrm{~B}), 2-5 \mathrm{~min}(5 \% \mathrm{~B} \rightarrow 50 \% \mathrm{~B}), 5-10 \mathrm{~min}(50 \% \mathrm{~B} \rightarrow 100 \% \mathrm{~B}), 10-15 \mathrm{~min}$ $(100 \% \mathrm{~B})$. The flow rate of the solvent was $1.0 \mathrm{~mL} / \mathrm{min}$, and the injection volume was $25 \mu \mathrm{L}$. All analysis was carried out at wavelength of $254 \mathrm{~nm}$ with a run time of $15 \mathrm{~min}$. HPLC-grade acetonitrile and water solvents were used. Acetic acid was added as a modifier to achieve a final concentration of $0.1 \%$ in each solvent.

Preparative HPLC purification was carried out using an Agilent 1100 HPLC system, $\mathrm{RP}-\mathrm{C} 18$ column $(250 \times 10 \mathrm{~mm}$; particle size $10 \mu \mathrm{m}$; Luna) with column oven temperature set at $25{ }^{\circ} \mathrm{C}$ and a gradient system of eluent water (A) and acetonitrile (B). The gradient condition was as follows: $0-2 \mathrm{~min}(5 \% \mathrm{~B}), 2-5 \mathrm{~min}(5 \% \mathrm{~B} \rightarrow 50 \% \mathrm{~B}), 5-10 \mathrm{~min}(50 \% \mathrm{~B} \rightarrow 100 \%$ 
B), 10-15 $\mathrm{min}(100 \% \mathrm{~B})$. The flow rate of the solvent was $3.0 \mathrm{~mL} / \mathrm{min}$, and the injection volume was $25 \mu \mathrm{L}$. All analysis was carried out at wavelength of $254 \mathrm{~nm}$ with a run time of 15 min. HPLC-grade acetonitrile and water solvents were used. Acetic acid was added as a modifier to achieve a final concentration of $0.1 \%$ in each solvent.

Other common chromatographic techniques, such as thin layer chromatography (TLC) on precoated silica gel $\mathrm{G}_{254}$ aluminum plates and silica gel flash column chromatography, were also engaged in the purification of the synthesized compounds.

\section{Experimental Methods}

3.1. General Method for Synthesis of (S,E)-11-[2-(Arylmethylene)hydrazono]-pyrrolo [2,1-c] [1,4] Benzodiazepines (4a-4q)

To a solution of $3(230 \mathrm{mg}, 1.0 \mathrm{mmol})$ in anhydrous methanol $(10 \mathrm{~mL})$ was added aldehyde ( $1.0 \mathrm{mmol}) .3 \AA$ molecular sieves $(0.5 \mathrm{~g})$ was also added and stirred at room temperature. Various modifications were used to obtain compounds $\mathbf{4 a - 4 q . ( S p e c t r o s c o p i c}$ data for PBD analogs (4a-4q) and typical MRM chromatograms of $4 \mathrm{k}$ in plasma and tissues are available in Supplementary Materials)

\section{2. (S,E)-11-[(1H-Pyrrol-2-yl)methylene)hydrazono]-pyrrolo[2,1-c][1,4] Benzodiazepine (4a)}

Starting material pyrrole-2-carboxaldehyde $(57.0 \mathrm{mg}, 0.60 \mathrm{mmol})$ was used and the reaction mixture was stirred under nitrogen gas overnight for $15 \mathrm{~h}$. Extraction was performed using chloroform/isopropanol (2:1) $(3 \times 20 \mathrm{~mL})$, where the organic layers were dried over anhydrous $\mathrm{Na}_{2} \mathrm{SO}_{4}$. The solvent mixture was then removed in vacuo and washed with diethyl ether, filtered off, and dried to afford a yellow solid of 4a. Yield $114.0 \mathrm{mg}(62.0 \%)$; ${ }^{1} \mathrm{H}-\mathrm{NMR}\left(400 \mathrm{MHz}, \mathrm{DMSO}-d_{6}\right): \delta=1.95(\mathrm{~m}, 3 \mathrm{H}), 2.78(\mathrm{bd}, 1 \mathrm{H}), 3.56(\mathrm{~m}, 2 \mathrm{H}), 4.43(\mathrm{bd}, 1 \mathrm{H}$, $\mathrm{H}-11 \mathrm{a}), 6.17(\mathrm{~s}, 1 \mathrm{H}), 6.51(\mathrm{~s}, 1 \mathrm{H}), 7.10(\mathrm{~s}, 1 \mathrm{H}), 7.19(\mathrm{~m}, 2 \mathrm{H}), 7.53(\mathrm{t}, J=8.0 \mathrm{~Hz}, 1 \mathrm{H}), 7.78(\mathrm{~d}$, $J=8.0 \mathrm{~Hz}, 1 \mathrm{H}), 8.21(\mathrm{~s}, 1 \mathrm{H}, \mathrm{CH}), 9.52(\mathrm{~s}, 1 \mathrm{H}, \mathrm{NH}) ;{ }^{13} \mathrm{C}-\mathrm{NMR}\left(100 \mathrm{MHz}, \mathrm{DMSO}-d_{6}\right): \delta=23.4$, 26.2, 47.3, 55.3 (C-11a), 109.9, 114.6, 121.9, 122.8, 123.3, 126.5, 128.9, 131.1, 132.6, 138.0, 147.1, $156.9,165.5(\mathrm{C}=\mathrm{O})$.

\section{3. (S,E)-11-[(1-Methyl-1H-imidazol-2-yl)methylene)hydrazono]-pyrrolo[2,1-c][1,4] Benzodiazepine (4b)}

Starting material 1-methylimidazole-2-carboxaldehyde $(95.8 \mathrm{mg}, 0.87 \mathrm{mmol})$ was used and the reaction mixture was stirred under nitrogen gas overnight for $15 \mathrm{~h}$. Extraction was performed using chloroform/isopropanol $(2: 1)(3 \times 20 \mathrm{~mL})$. The combined organic layers were dried over anhydrous $\mathrm{Na}_{2} \mathrm{SO}_{4}$. The solvent mixture was then removed in vacuo and washed with diethyl ether, filtered off, and dried to afford an off-white solid of $4 \mathbf{b}$. Yield $182.0 \mathrm{mg}(65.0 \%) ;{ }^{1} \mathrm{H}-\mathrm{NMR}\left(400 \mathrm{MHz}, \mathrm{DMSO}-d_{6}\right): \delta=1.98(\mathrm{~m}, 3 \mathrm{H}), 2.81(\mathrm{bd}, 1 \mathrm{H}), 3.56(\mathrm{~m}$, 2H), $4.03(\mathrm{~s}, 3 \mathrm{H}), 4.43(\mathrm{bd}, 1 \mathrm{H}, \mathrm{H}-11 \mathrm{a}), 7.09(\mathrm{~s}, 1 \mathrm{H}), 7.17(\mathrm{~m}, 1 \mathrm{H}), 7.33(\mathrm{~d}, J=8.0 \mathrm{~Hz}, 1 \mathrm{H})$, 7.39 (s, 1H), $7.48(\mathrm{bt}, 1 \mathrm{H}), 7.77(\mathrm{~d}, J=8.0 \mathrm{~Hz}, 1 \mathrm{H}), 8.30(\mathrm{~s}, 1 \mathrm{H}, \mathrm{CH}) ;{ }^{13} \mathrm{C}-\mathrm{NMR}(100 \mathrm{MHz}$, DMSO- $\left.d_{6}\right): \delta=23.3,26.5,35.7,47.4,55.3$ (C-11a), 122.6, 123.7, 126.2, 126.6, 129.7, 130.8, 131.1, $132.4,137.6,142.3,156.7,165.3(\mathrm{C}=\mathrm{O})$.

\section{4. (S,E)-11-[(Thiazol-5-ylmethylene)hydrazono]-pyrrolo[2,1-c][1,4] Benzodiazepine (4c)}

Starting material 5-formylthiazole $(56.6 \mathrm{mg}, 0.60 \mathrm{mmol})$ was used and the reaction mixture was stirred under nitrogen gas overnight for $15 \mathrm{~h}$. Extraction was performed using chloroform/isopropanol (2:1) $(3 \times 20 \mathrm{~mL})$, where the organic layers were dried over anhydrous $\mathrm{Na}_{2} \mathrm{SO}_{4}$. The solvent mixture was then removed in vacuo and washed with diethyl ether, filtered off, and dried to afford a yellow solid of 4c. Yield $115.5 \mathrm{mg}(71.0 \%)$; ${ }^{1} \mathrm{H}-\mathrm{NMR}\left(400 \mathrm{MHz}, \mathrm{DMSO}-d_{6}\right): \delta=2.08(\mathrm{~m}, 3 \mathrm{H}), 3.05(\mathrm{bd}, 1 \mathrm{H}), 3.59(\mathrm{~m}, 2 \mathrm{H}), 4.49(\mathrm{bd}$, $1 \mathrm{H}, \mathrm{H}-11 \mathrm{a}), 7.19$ (t, J = 7.2 Hz, 1H), $7.37(\mathrm{~d}, J=8.0 \mathrm{~Hz}, 1 \mathrm{H}), 7.48(\mathrm{t}, J=7.2 \mathrm{~Hz}, 1 \mathrm{H}), 7.76$ $(\mathrm{d}, J=8.0 \mathrm{~Hz}, 1 \mathrm{H}), 8.36(\mathrm{~s}, 1 \mathrm{H}), 8.43(\mathrm{~s}, 1 \mathrm{H}), 9.26(\mathrm{~s}, 1 \mathrm{H}, \mathrm{CH}), 9.88(\mathrm{~s}, 1 \mathrm{H}, \mathrm{NH}) ;{ }^{13} \mathrm{C}-\mathrm{NMR}$ $\left(100 \mathrm{MHz}, \mathrm{DMSO}-d_{6}\right): \delta=23.7,26.5,47.4,55.8$ (C-11a), 122.9, 123.8, 126.9, 127.8, 130.7, 132.4, $137.5,144.7,149.2,158.2,160.6,165.3(\mathrm{C}=\mathrm{O})$. 


\section{5. (S,E)-11-[(2-Methylthiazol-5-yl)methylene)hydrazono]-pyrrolo[2,1-c][1,4] Benzodiazepine (4d)}

Starting material 2-methylthiazole-5-carboxaldehyde (166.0 mg, $1.305 \mathrm{mmol}$ ) was used and the reaction mixture was stirred under nitrogen gas overnight for $15 \mathrm{~h}$. Extraction was performed using chloroform/isopropanol $(2: 1)(3 \times 20 \mathrm{~mL})$, where the organic layers were dried over anhydrous $\mathrm{Na}_{2} \mathrm{SO}_{4}$. The solvent mixture was then removed in vacuo and washed with diethyl ether, filtered off, and dried to afford a yellow solid of $4 \mathbf{d d}$. Yield $319.0 \mathrm{mg}(72.0 \%) ;{ }^{1} \mathrm{H}-\mathrm{NMR}\left(400 \mathrm{MHz}, \mathrm{DMSO}-d_{6}\right): \delta=2.01(\mathrm{~m}, 3 \mathrm{H}), 3.04(\mathrm{bd}, 1 \mathrm{H}), 3.37(\mathrm{~s}$, $3 \mathrm{H}), 3.56(\mathrm{~m}, 2 \mathrm{H}), 4.47(\mathrm{bd}, 1 \mathrm{H}, \mathrm{H}-11 \mathrm{a}), 7.18(\mathrm{t}, J=7.4 \mathrm{~Hz}, 1 \mathrm{H}), 7.36(\mathrm{~d}, J=7.4 \mathrm{~Hz}, 1 \mathrm{H}), 7.47$ $(\mathrm{t}, J=7.4 \mathrm{~Hz}, 1 \mathrm{H}), 7.76(\mathrm{~d}, J=7.4 \mathrm{~Hz}, 1 \mathrm{H}), 8.17(\mathrm{~s}, 1 \mathrm{H}), 8.23(\mathrm{~s}, 1 \mathrm{H}, \mathrm{CH}) ;{ }^{13} \mathrm{C}-\mathrm{NMR}(100 \mathrm{MHz}$, DMSO- $\left.d_{6}\right): \delta=19.3,23.8,26.5,47.4,55.7$ (C-11a), 122.8, 123.8, 126.9, 128.0, 130.8, 132.3, 137.5, 145.0, 148.6, 158.1, $165.2(\mathrm{C}=\mathrm{O}), 172.3$.

\section{6. (S,E)-11-[(4-Methylthiazol-5-yl)methylene)hydrazono]-pyrrolo[2,1-c][1,4] Benzodiazepine (4e)}

Starting material 4-methylthiazole-5-carboxaldehyde (122.0 mg, $0.957 \mathrm{mmol}$ ) was used and the reaction mixture was stirred under nitrogen gas overnight for $15 \mathrm{~h}$. Extraction was performed using chloroform/isopropanol $(2: 1)(3 \times 20 \mathrm{~mL})$, where the organic layers were dried over anhydrous $\mathrm{Na}_{2} \mathrm{SO}_{4}$. The solvent mixture was then removed in vacuo and washed with diethyl ether, filtered off, and dried to afford an off-white solid of 4e. Yield $198.0 \mathrm{mg}(61.0 \%) ;{ }^{1} \mathrm{H}-\mathrm{NMR}\left(400 \mathrm{MHz}, \mathrm{DMSO}-d_{6}\right): \delta=2.01(\mathrm{~m}, 3 \mathrm{H}), 3.04(\mathrm{bd}, 1 \mathrm{H}), 3.37(\mathrm{~s}$, $3 \mathrm{H}), 3.56(\mathrm{~m}, 2 \mathrm{H}), 4.47(\mathrm{bd}, 1 \mathrm{H}, \mathrm{H}-11 \mathrm{a}), 7.18(\mathrm{~m}, 1 \mathrm{H}), 7.36(\mathrm{~m}, 1 \mathrm{H}), 7.47(\mathrm{~m}, 1 \mathrm{H}), 8.25(\mathrm{~s}, 1 \mathrm{H})$, $8.67(\mathrm{~s}, 1 \mathrm{H}, \mathrm{CH}) ;{ }^{13} \mathrm{C}-\mathrm{NMR}\left(100 \mathrm{MHz}, \mathrm{DMSO}-d_{6}\right): \delta=16.3,23.5,26.2,47.4,55.8$ (C-11a), $122.8,123.8,126.9,128.5,130.7,132.3,137.5,144.3,149.2,155.7,158.1,165.3(\mathrm{C}=\mathrm{O})$.

\section{7. (S,E)-11-[(2-Aminothiazol-5-yl)methylene)hydrazono]-pyrrolo[2,1-c][1,4] Benzodiazepine (4f)}

Starting material 2-aminothiazole-5-carboxaldehyde $(64.0 \mathrm{mg}, 0.50 \mathrm{mmol})$ was used and the reaction mixture was stirred under nitrogen gas overnight for $15 \mathrm{~h}$. Extraction was performed using chloroform/isopropanol $(2: 1)(3 \times 20 \mathrm{~mL})$, where the organic layers were dried over anhydrous $\mathrm{Na}_{2} \mathrm{SO}_{4}$. The solvent mixture was then removed in vacuo and washed with diethyl ether, filtered off, and dried to afford an off-white solid of $\mathbf{4 f}$. Yield $115.6 \mathrm{mg}(68.0 \%) .{ }^{1} \mathrm{H}-\mathrm{NMR}\left(400 \mathrm{MHz}, \mathrm{DMSO}-d_{6}\right): \delta=2.05(\mathrm{~m}, 3 \mathrm{H}), 3.03(\mathrm{bs}, 1 \mathrm{H})$, $3.56(\mathrm{~m}, 2 \mathrm{H}), 4.43(\mathrm{bd}, 1 \mathrm{H}, \mathrm{H}-11 \mathrm{a}), 7.14(\mathrm{t}, J=7.5 \mathrm{~Hz}, 1 \mathrm{H}), 7.34(\mathrm{~d}, J=7.5 \mathrm{~Hz}, 1 \mathrm{H}), 7.45$ $(\mathrm{d}, J=7.5 \mathrm{~Hz}, 1 \mathrm{H}), 7.73(\mathrm{~d}, J=7.5 \mathrm{~Hz}, 1 \mathrm{H}), 7.91(\mathrm{~s}, 1 \mathrm{H}, \mathrm{CH}), 9.55(\mathrm{~s}, 1 \mathrm{H}, \mathrm{NH}) ;{ }^{13} \mathrm{C}-\mathrm{NMR}$ $\left(100 \mathrm{MHz}, \mathrm{DMSO}-d_{6}\right): \delta=23.6,26.6,47.2,55.7$ (C-11a), 117.3, 122.5, 123.3, 126.5, 130.7, 132.3, $138.0,146.6,148.6,156.2,165.4(\mathrm{C}=\mathrm{O}), 175.5$.

\section{8. (S,E)-11-[(2,4-Dichlorothiazol-5-yl)methylene)hydrazono]-pyrrolo[2,1-c][1,4] Benzodiazepine (4g)}

Starting material 2,4-dichlorothiazole-5-carboxaldehyde (182.0 mg, $1.0 \mathrm{mmol})$ was used and the reaction mixture was stirred under nitrogen gas overnight for $15 \mathrm{~h}$. Extraction was performed using chloroform/isopropanol $(2: 1)(3 \times 20 \mathrm{~mL})$, where the organic layers were dried over anhydrous $\mathrm{Na}_{2} \mathrm{SO}_{4}$. The solvent mixture was then removed in vacuo and washed with diethyl ether, filtered off, and dried to afford an off-white solid of $4 \mathrm{~g}$. Yield $272.0 \mathrm{mg}(69.0 \%) ;{ }^{1} \mathrm{H}-\mathrm{NMR}\left(400 \mathrm{MHz}, \mathrm{DMSO}-\mathrm{d}_{6}\right): \delta=2.01(\mathrm{~m}, 3 \mathrm{H}), 2.89(\mathrm{bd}, 1 \mathrm{H}), 3.58(\mathrm{~m}$, 2H), 4.50 (bs, 1H, H-11a), 7.21 (s, 1H), 7.39 (s, 1H), $7.48(\mathrm{bs}, 1 \mathrm{H}), 7.78(\mathrm{~s}, 1 \mathrm{H}), 8.07(\mathrm{~s}, 1 \mathrm{H}$, $\mathrm{CH}), 10.17(\mathrm{~s}, 1 \mathrm{H}, \mathrm{NH}) ;{ }^{13} \mathrm{C}-\mathrm{NMR}\left(100 \mathrm{MHz} \mathrm{CDCl}_{3}\right): \delta=23.5,26.8,47.3,55.6,121.9,123.2$, $124.4,127.2,130.6,132.4,137.1,139.4,141.0,156.6,160.3,165.1(\mathrm{C}=\mathrm{O})$.

\section{9. (S,E)-11-[2-(Benzo[b]thiophenylmethylene)hydrazono]-pyrrolo[2,1-c][1,4] Benzodiazepine (4h)}

Starting material benzo[b]thiophene-2-carbaldehyde (100.0 mg, $0.61 \mathrm{mmol})$ was used and the reaction mixture was stirred under nitrogen gas overnight for $15 \mathrm{~h}$. Extraction was performed using chloroform/isopropanol (2:1) $(3 \times 20 \mathrm{~mL})$. The combined organic layers were dried over anhydrous $\mathrm{Na}_{2} \mathrm{SO}_{4}$. The solvent mixture was then removed in vacuo and washed with diethyl ether, filtered off, and dried to afford an off-white solid of $4 \mathbf{h}$. Yield $172.0 \mathrm{mg}(75.0 \%) ;{ }^{1} \mathrm{H}-\mathrm{NMR}\left(400 \mathrm{MHz}, \mathrm{CDCl}_{3}\right): \delta=2.02-2.07(\mathrm{~m}, 3 \mathrm{H}), 2.98-3.02(\mathrm{~m}, 1 \mathrm{H})$, 
3.68-3.76 (m, 1H), 3.80-3.87 (m, 1H), 4.39 (d, J = 7.6 Hz, 1H, H-11a), $7.04(\mathrm{~d}, J=7.3 \mathrm{~Hz}, 1 \mathrm{H})$, $7.22(\mathrm{t}, J=7.3 \mathrm{~Hz}, 1 \mathrm{H}), 7.40(\mathrm{~m}, 2 \mathrm{H}), 7.47(\mathrm{~m}, 1 \mathrm{H}), 7.59(\mathrm{~s}, 1 \mathrm{H}), 7.79-7.86(\mathrm{~m}, 2 \mathrm{H}), 8.00(\mathrm{~d}$, $J=7.6 \mathrm{~Hz}, 1 \mathrm{H}), 8.53(\mathrm{~s}, 1 \mathrm{H}, \mathrm{CH}), 8.71(\mathrm{~s}, 1 \mathrm{H}, \mathrm{NH}) ;{ }^{13} \mathrm{C}-\mathrm{NMR}\left(100 \mathrm{MHz} \mathrm{CDCl}_{3}\right): \delta=23.5$, 25.9, 47.4, 55.4 (C-11a), 120.8, 122.6, 123.9, 124.4, 124.7, 126.2, 126.4, 128.4, 131.4, 132.4, 136.6, $139.4,139.9,140.6,151.6,157.8,165.9(\mathrm{C}=\mathrm{O})$.

\subsection{0. (S,E)-11-[(Benzo[b]thiophen-2-ylmethylene)hydrazono]-7-bromo-pyrrolo[2,1-c][1,4] Benzodiazepine $(\mathbf{4 i})$}

Starting material benzothiophene-2-carboxaldehyde $(81.0 \mathrm{mg}, 0.50 \mathrm{mmol})$ was used and the reaction mixture was stirred under nitrogen gas overnight for $15 \mathrm{~h}$. Extraction was performed using chloroform/isopropanol $(2: 1)(3 \times 20 \mathrm{~mL})$, where the organic layers were dried over anhydrous $\mathrm{Na}_{2} \mathrm{SO}_{4}$. The solvent mixture was then removed in vacuo and washed with diethyl ether, filtered off, and dried to afford an off-white solid of 4i. Yield $158.6 \mathrm{mg}(70.0 \%) ;{ }^{1} \mathrm{H}-\mathrm{NMR}\left(400 \mathrm{MHz}, \mathrm{DMSO}-d_{6}\right): \delta=2.00(\mathrm{~m}, 3 \mathrm{H}), 2.78(\mathrm{bs}, 1 \mathrm{H}), 3.58(\mathrm{~m}$, 2H), 4.48 (bd, 1H, H-11a), $7.41(\mathrm{~m}, 1 \mathrm{H}), 7.68(\mathrm{dd}, J=2.3,8.8 \mathrm{~Hz}, 1 \mathrm{H}), 7.84(\mathrm{~d}, J=2.3 \mathrm{~Hz}$, 1H), 7.89 (m, 2H), 7.98 (bd, 1H), 8.75 (s, 1H, CH), 9.31 (s, 1H, NH); ${ }^{13} \mathrm{C}-\mathrm{NMR}(100 \mathrm{MHz}$, DMSO- $\left.d_{6}\right): \delta=23.5,26.3,47.4,55.3$ (C-11a), 115.5, 123.1, 125.0, 125.1, 125.3, 126.7, 128.4, $129.4,132.9,134.9,137.1,139.6,140.2,140.5,152.2,156.4,164.0(\mathrm{C}=\mathrm{O})$.

\subsection{1. (S,E)-11-[(Benzo[b]thiophen-3-ylmethylene)hydrazono]-7-bromo-pyrrolo[2,1-c][1,4] Benzodiazepine (4j)}

Starting material benzothiophene-3-carboxaldehyde $(81.0 \mathrm{mg}, 0.50 \mathrm{mmol})$ was used and the reaction mixture was stirred under nitrogen gas overnight for $15 \mathrm{~h}$. Extraction was performed using chloroform/isopropanol $(2: 1)(3 \times 20 \mathrm{~mL})$, where the organic layers were dried over anhydrous $\mathrm{Na}_{2} \mathrm{SO}_{4}$. The solvent mixture was then removed in vacuo and washed with diethyl ether, filtered off, and dried to afford an off-white solid of $4 \mathbf{j}$. Yield $163.0 \mathrm{mg}(72.0 \%) .{ }^{1} \mathrm{H}-\mathrm{NMR}\left(400 \mathrm{MHz}, \mathrm{DMSO}-d_{6}\right): \delta=1.97(\mathrm{~m}, 3 \mathrm{H}), 2.83$ (bs, $\left.1 \mathrm{H}\right), 3.59(\mathrm{~m}$, 2H), 4.53 (bd, 1H, H-11a), 7.38 (d, $J=8.6 \mathrm{~Hz} 1 \mathrm{H}), 7.49(\mathrm{~m}, 2 \mathrm{H}), 7.68(\mathrm{~d}, J=8.6 \mathrm{~Hz}, 1 \mathrm{H}), 7.84$ (bs, $1 \mathrm{H}), 8.06(\mathrm{~d}, J=7.5 \mathrm{~Hz}, 1 \mathrm{H}), 8.46(\mathrm{~s}, 1 \mathrm{H}), 8.75(\mathrm{bd}, 1 \mathrm{H}), 8.77(\mathrm{~s}, 1 \mathrm{H}, \mathrm{CH}), 9.26(\mathrm{~s}, 1 \mathrm{H}, \mathrm{NH})$; ${ }^{13} \mathrm{C}-\mathrm{NMR}\left(100 \mathrm{MHz}, \mathrm{DMSO}-d_{6}\right): \delta=23.7,26.4,47.6,55.6$ (C-11a), 115.3, 123.3, 125.1, 125.5, $125.6,125.7,128.4,132.1,132.9,133.9,134.9,136.5,137.2,140.4,152.9,155.7,164.1(\mathrm{C}=\mathrm{O})$.

\subsection{2. (S,E)-11-[(Benzo[b]thiophen-2-ylmethylene)hydrazono]-8-chloro-pyrrolo[2,1-c][1,4] Benzodiazepine (4k)}

Starting material benzothiophene-2-carboxaldehyde $(81.0 \mathrm{mg}, 0.50 \mathrm{mmol})$ was used and the reaction mixture was stirred under nitrogen gas overnight for $15 \mathrm{~h}$. Extraction was performed using chloroform/isopropanol $(2: 1)(3 \times 20 \mathrm{~mL})$, where the organic layers were dried over anhydrous $\mathrm{Na}_{2} \mathrm{SO}_{4}$. The solvent mixture was then removed in vacuo and washed with diethyl ether, filtered off, and dried to afford an off-white solid of $4 \mathbf{k}$. Yield $153.0 \mathrm{mg}(75.0 \%) ;{ }^{1} \mathrm{H}-\mathrm{NMR}\left(400 \mathrm{MHz}, \mathrm{DMSO}-d_{6}\right): \delta=1.99(\mathrm{~m}, 3 \mathrm{H}), 2.79(\mathrm{bs}, 1 \mathrm{H})$, $3.58(\mathrm{~m}, 2 \mathrm{H}), 4.49$ (bd, 1H, H-11a), 7.23 (d, J = 8.5 Hz, 1H), $7.42(\mathrm{~m}, 2 \mathrm{H}), 7.60$ (bs, 1H), 7.77 $(\mathrm{d}, J=8.5 \mathrm{~Hz}, 1 \mathrm{H}), 7.94(\mathrm{~m}, 3 \mathrm{H}), 8.75(\mathrm{~s}, 1 \mathrm{H}, \mathrm{CH}), 9.34(\mathrm{~s}, 1 \mathrm{H}, \mathrm{NH}) ;{ }^{13} \mathrm{C}-\mathrm{NMR}(100 \mathrm{MHz}$, DMSO- $\left.d_{6}\right): \delta=23.3,26.3,47.4,55.3$ (C-11a), 122.1, 123.1, 123.5, 125.0, 125.2, 125.4, 126.6, $129.4,132.8,136.5,138.9,139.6,140.3,140.6,152.3,156.3,164.5$ (C=O).

\subsection{3. (S,E)-11-[(3-Methylbenzo[b]thiophen-2-yl)methylene)hydrazono]-pyrrolo[2,1-c][1,4] Benzodiazepine (41)}

Starting material 3-methylbenzo[b]thiophene-2-carboxaldehyde (106.0 mg, $0.60 \mathrm{mmol})$ was used and the reaction mixture was stirred under nitrogen gas overnight for $15 \mathrm{~h}$. Extraction was performed using chloroform/isopropanol $(2: 1)(3 \times 20 \mathrm{~mL})$, where the organic layers were dried over anhydrous $\mathrm{Na}_{2} \mathrm{SO}_{4}$. The solvent mixture was then removed in vacuo and washed with diethyl ether, filtered off, and dried to afford a yellow solid of 41. Yield $152.0 \mathrm{mg}(65.0 \%) ;{ }^{1} \mathrm{H}-\mathrm{NMR}\left(400 \mathrm{MHz}, \mathrm{DMSO}-d_{6}\right): \delta=2.00(\mathrm{~m}, 3 \mathrm{H}), 2.82(\mathrm{bs}, 1 \mathrm{H}), 3.37$ $(\mathrm{s}, 3 \mathrm{H}), 3.58(\mathrm{~m}, 2 \mathrm{H}), 4.44(\mathrm{bd}, 1 \mathrm{H}, \mathrm{H}-11 \mathrm{a}), 7.19(\mathrm{t}, J=7.0 \mathrm{~Hz}, 1 \mathrm{H}), 7.44(\mathrm{~m}, 4 \mathrm{H}), 7.84(\mathrm{~m}, 3 \mathrm{H})$, 8.85 (s, 1H, CH), 9.01 (s, 1H, NH) ${ }^{13} \mathrm{C}-\mathrm{NMR}\left(100 \mathrm{MHz}, \mathrm{DMSO}-d_{6}\right): \delta=11.8,23.5,26.3,47.2$, 
55.3 (C-11a), 122.5, 122.9, 123.1, 123.7, 124.9, 126.6, 126.9, 130.8, 132.5, 134.4, 136.4, 137.4, $139.9,140.6,150.6,156.9,165.3(\mathrm{C}=\mathrm{O})$.

\subsection{4. (S,E)-11-[(3-Chlorobenzo[b]thiophen-2-yl)methylene)hydrazono]-pyrrolo[2,1-c][1,4] Benzodiazepine (4m)}

Starting material 3-chloro-1-benzothiophene-2-carboxaldehyde $(98.4 \mathrm{mg}, 0.60 \mathrm{mmol})$ was used and the reaction mixture was stirred under nitrogen gas overnight for $15 \mathrm{~h}$. Extraction was performed using chloroform/isopropanol $(2: 1)(3 \times 20 \mathrm{~mL})$, where the organic layers were dried over anhydrous $\mathrm{Na}_{2} \mathrm{SO}_{4}$. The solvent mixture was then removed in vacuo and washed with diethyl ether, filtered off, and dried to afford an off-white solid of 4m. Yield $122.7 \mathrm{mg}(60.0 \%) ;{ }^{1} \mathrm{H}-\mathrm{NMR}\left(400 \mathrm{MHz}, \mathrm{DMSO}-d_{6}\right): \delta=2.12(\mathrm{~m}, 3 \mathrm{H}), 3.16(\mathrm{bs}, 1 \mathrm{H})$, $3.61(\mathrm{~m}, 2 \mathrm{H}), .4 .52(\mathrm{bd}, 1 \mathrm{H}, \mathrm{H}-11 \mathrm{a}), 7.21(\mathrm{t}, J=7.2 \mathrm{~Hz}, 1 \mathrm{H}), 7.39(\mathrm{~m}, 1 \mathrm{H}), 7.54(\mathrm{~m}, 2 \mathrm{H}), 7.78$ $(\mathrm{d}, J=7.2 \mathrm{~Hz}, 1 \mathrm{H}), 7.89(\mathrm{~m}, 1 \mathrm{H}), 8.07(\mathrm{~m}, 1 \mathrm{H}), 8.39(\mathrm{~s}, 1 \mathrm{H}, \mathrm{CH}), 9.98(\mathrm{~s}, 1 \mathrm{H}, \mathrm{NH}) ;{ }^{13} \mathrm{C}-\mathrm{NMR}$ $\left(100 \mathrm{MHz}, \mathrm{DMSO}-d_{6}\right): \delta=23.8,26.9,47.4,55.8$ (C-11a), 122.5, 122.9, 123.4, 124.0, 124.3, 125.9, $126.2,127.0,128.1,130.8,132.5,134.6,137.4,140.8,142.9,158.9,165.2(\mathrm{C}=\mathrm{O})$.

\subsection{5. (S,E)-11-[(Benzo[d]thiazol-2-ylmethylene)hydrazono]-pyrrolo[2,1-c][1,4] Benzodiazepine (4n)}

Starting material 1,3-benzothiazole-2-carboxaldehyde $(142.0 \mathrm{mg}, 0.87 \mathrm{mmol})$ was used and the reaction mixture was stirred under nitrogen gas overnight for $15 \mathrm{~h}$. Extraction was performed using chloroform/isopropanol $(2: 1)(3 \times 20 \mathrm{~mL})$, where the organic layers were dried over anhydrous $\mathrm{Na}_{2} \mathrm{SO}_{4}$. The solvent mixture was then removed in vacuo and washed with diethyl ether, filtered off, and dried to afford a yellow solid of $4 \mathbf{n}$. Yield 222.0 $\mathrm{mg}(68.0 \%) ;{ }^{1} \mathrm{H}-\mathrm{NMR}\left(400 \mathrm{MHz}, \mathrm{DMSO}-d_{6}\right): \delta=1.97(\mathrm{~m}, 3 \mathrm{H}), 2.80(\mathrm{bd}, 1 \mathrm{H}), 3.57(\mathrm{~m}, 2 \mathrm{H})$, 4.50 (bd, 1H, H-11a), $7.40(\mathrm{~m}, 3 \mathrm{H}), 7.68(\mathrm{dd}, J=8.9,2.2 \mathrm{~Hz}, 1 \mathrm{H}), 7.84(\mathrm{~d}, J=2.2 \mathrm{~Hz}, 1 \mathrm{H}), 7.90$ $(\mathrm{m}, 2 \mathrm{H}), 7.98(\mathrm{bd}, 1 \mathrm{H}), 8.75(\mathrm{~s}, 1 \mathrm{H}, \mathrm{CH}), 9.30(\mathrm{~s}, 1 \mathrm{H}, \mathrm{NH}) ;{ }^{13} \mathrm{C}-\mathrm{NMR}\left(100 \mathrm{MHz}, \mathrm{DMSO}-d_{6}\right)$ : $\delta=23.4,26.3,47.4,55.3$ (C-11a) , 115.4, 123.1, 124.9, 124.4, 125.2, 126.7, 128.5, 129.4, 132.9, $134.9,137.0,139.6,140.3,140.6,152.1,156.4,164.0(\mathrm{C}=\mathrm{O})$.

\subsection{6. (S,E)-11-[(1-Methyl-1H-indol-3-yl)methylene)hydrazono]-pyrrolo[2,1-c][1,4] Benzodiazepine (4o)}

Starting material 1-methylindole-3-carboxaldehyde (152.0 mg, $0.957 \mathrm{mmol}$ ) was used and the reaction mixture was stirred under nitrogen gas overnight for $15 \mathrm{~h}$. The combined organic layers were dried over anhydrous $\mathrm{Na}_{2} \mathrm{SO}_{4}$. The solvent mixture was then removed in vacuo and washed with diethyl ether, filtered off, and dried to afford a yellow solid of 4o. Yield $244.0 \mathrm{mg}(69.0 \%) ;{ }^{1} \mathrm{H}-\mathrm{NMR}\left(400 \mathrm{MHz}, \mathrm{DMSO}-d_{6}\right): \delta=1.98(\mathrm{~m}, 3 \mathrm{H}), 2.87(\mathrm{bs}, 1 \mathrm{H})$, $3.36(\mathrm{~s}, 3 \mathrm{H}), 3.58(\mathrm{~m}, 2 \mathrm{H}), 4.45$ (bs, 1H, H-11a), $7.23(\mathrm{~m}, 3 \mathrm{H}), 7.34(\mathrm{bd}, 1 \mathrm{H}), 7.51(\mathrm{~m}, 2 \mathrm{H}), 7.78$ $(\mathrm{d}, J=8.0 \mathrm{~Hz}, 1 \mathrm{H}), 7.92(\mathrm{~s}, 1 \mathrm{H}), 8.38(\mathrm{~d}, J=8.0 \mathrm{~Hz}, 1 \mathrm{H}), 8.65(\mathrm{~s}, 1 \mathrm{H}, \mathrm{CH}), 8.98(\mathrm{~s}, 1 \mathrm{H}, \mathrm{NH})$; ${ }^{13} \mathrm{C}-\mathrm{NMR}\left(100 \mathrm{MHz}, \mathrm{DMSO}-d_{6}\right): \delta=23.5,26.2,33.3,47.3,55.5$ (C-11a), 55.6, 110.6, 111.7, $121.4,122.3,122.9,123.1,125.6,126.4,130.9,132.4,132.5,135.8,138.0,138.1,153.3,154.7$, $165.5(\mathrm{C}=\mathrm{O})$.

\subsection{7. (S,E)-11-[(5-Methoxy-1H-indol-3-yl)methylene)hydrazono]-pyrrolo[2,1-c][1,4] Benzodiazepine (4p)}

Starting material 5-methoxy-1-methylindole-3-carbaldehyde (100.0 mg, $0.57 \mathrm{mmol})$ was used and the reaction mixture was stirred under nitrogen gas overnight for $15 \mathrm{~h}$. Extraction was performed using chloroform/isopropanol $(2: 1)(3 \times 20 \mathrm{~mL})$. The combined organic layers were dried over anhydrous $\mathrm{Na}_{2} \mathrm{SO}_{4}$. The solvent mixture was then removed in vacuo and washed with diethyl ether, filtered off, and dried to afford a brown solid of 4p. Yield $153.0 \mathrm{mg}(68.0 \%) ;{ }^{1} \mathrm{H}-\mathrm{NMR}\left(400 \mathrm{MHz}, \mathrm{DMSO}-d_{6}\right): \delta=1.99(\mathrm{~m}, 3 \mathrm{H}), 2.84(\mathrm{bs}, 1 \mathrm{H})$, $3.37(\mathrm{~s}, 3 \mathrm{H}), 3.60(\mathrm{~m}, 2 \mathrm{H}), 4.47$ (bs, 1H, H-11a), $6.84(\mathrm{bd}, 1 \mathrm{H}), 7.14(\mathrm{bt}, 1 \mathrm{H}), 7.34(\mathrm{~m}, 2 \mathrm{H}), 7.49$ (bt, 1H), $7.77(\mathrm{bd}, 1 \mathrm{H}), 7.80(\mathrm{~d}, J=2.4 \mathrm{~Hz}, 1 \mathrm{H}), 7.89(\mathrm{~d}, J=2.4 \mathrm{~Hz}, 1 \mathrm{H}), 8.67(\mathrm{~s}, 1 \mathrm{H}, \mathrm{CH}), 9.01$ (s, 1H, NH); ${ }^{13} \mathrm{C}-\mathrm{NMR}\left(100 \mathrm{MHz}, \mathrm{DMSO}-d_{6}\right): \delta=23.6,26.2,47.4,55.4$ (C-11a), 55.6, 104.0, $112.4,112.9,113.0,121.7,123.0,125.8,126.0,131.0,132.3,132.4,132.6,138.1,153.7,154.6$, $154.9,165.5(\mathrm{C}=\mathrm{O})$. 


\subsection{8. (S,E)-11-[(Adamantan-1-yl)methylene)hydrazono]-pyrrolo[2,1-c][1,4] Benzodiazepine (4q)}

Starting material adamantane-1-carboxaldehyde (164.0 mg, $1.0 \mathrm{mmol})$ was used and the reaction mixture was stirred under nitrogen gas overnight for $15 \mathrm{~h}$. Extraction was performed using chloroform/isopropanol $(2: 1)(3 \times 20 \mathrm{~mL})$, where the organic layers were dried over anhydrous $\mathrm{Na}_{2} \mathrm{SO}_{4}$. The solvent mixture was then removed in vacuo and washed with diethyl ether, filtered off, and dried to afford a yellow solid of 4q. Yield 256.0 $\operatorname{mg}(68.0 \%) ;{ }^{1} \mathrm{H}-\mathrm{NMR}\left(400 \mathrm{MHz}, \mathrm{CDCl}_{3}\right): \delta=1.74(\mathrm{~m}, 11 \mathrm{H}), 2.01(\mathrm{~m}, 7 \mathrm{H}), 2.91(\mathrm{bd}, 1 \mathrm{H})$, $3.75(\mathrm{~m}, 2 \mathrm{H}), 4.29(\mathrm{bd}, 1 \mathrm{H}, \mathrm{H}-11 \mathrm{a}), 6.95(\mathrm{~d}, J=9.5 \mathrm{~Hz}, 1 \mathrm{H}), 7.15(\mathrm{t}, J=8.4 \mathrm{~Hz}, 1 \mathrm{H}), 7.41$ $(\mathrm{t}, J=7.4 \mathrm{~Hz}, 1 \mathrm{H}), 7.95(\mathrm{~d}, J=8.4 \mathrm{~Hz}, 1 \mathrm{H}), 8.40(\mathrm{~s}, 1 \mathrm{H}, \mathrm{CH}) ;{ }^{13} \mathrm{C}-\mathrm{NMR}\left(100 \mathrm{MHz}, \mathrm{CDCl}_{3}\right)$ : $\delta=23.4,26.0,27.9,36.7,37.3,39.7,47.3,50.6,55.3$ (C-11a), 120.6, 123.5, 126.1, 131.3, 132.3, $136.9,156.9,166.1,168.9(\mathrm{C}=\mathrm{O})$.

\section{Cannabinoid Receptor Binding Assay}

The affinities of the compounds for CB1 and CB2 receptors were examined using displacement assays, as previously described [13]. Briefly, cell membranes from $\mathrm{CHO}$ cells expressing human $\mathrm{CB} 1$ or human $\mathrm{CB} 2$ receptors were isolated using differential centrifugation. Test compounds reconstituted in DMSO and were incubated with the isolated membrane in binding buffer ( $50 \mathrm{mM}$ Tris- $\mathrm{HCl}, 1 \mathrm{mM}$ EDTA, $3 \mathrm{mM} \mathrm{MgCl}_{2}, 5 \mathrm{mg} / \mathrm{mL}$ BSA, pH 7.4) along with $2.5 \mathrm{nM}\left[{ }^{3} \mathrm{H}\right] \mathrm{CP}-55,940$. Total binding was assessed in the presence of equal concentration of DMSO, while non-specific binding was determined in the presence of $10 \mu \mathrm{M} \mathrm{CP}-55,940$, and background binding was determined in wells lacking membrane. Following incubation at $30^{\circ} \mathrm{C}$ for $60 \mathrm{~min}$, the binding reactions were terminated by vacuum filtration through Whatman GF/C filters. The filters were then washed twice with ice-cold buffer (50 mM Tris-HCl, $1 \mathrm{mg} / \mathrm{mL}$ BSA). Liquid scintillation cocktail was added to each well and the total tritiated counts per minute were analyzed using a TopCount scintillation counter. Background counts were subtracted from all wells and the percent displacement from total binding was calculated.

The compounds were initially screened at $10 \mu \mathrm{M}$ concentrations. If they produced at least $\pm 30 \%$ displacement of the radioligand, then full competition curves were constructed. $K_{i}$ values were calculated using GraphPad Prism (San Diego, CA, USA) and $K_{\mathrm{d}}$ values determined using a 1 site fit. All assays were run in technical and biological replicates so that the $n=5-6$.

\section{PK Evaluation in CD-1 Mice}

5.1. Preparation of Stock Solutions, Calibration Standards, Quality Control Samples and Internal Standard Solution

The primary stock solutions of $\mathbf{4 k}$ and phenacetin (internal standard, IS) were prepared in methanol at a concentration of $1.0 \mathrm{mg} / \mathrm{mL}$. Working solutions of calibration standards and quality control (QC) samples were prepared by dilution with methanol and stored at $-20^{\circ} \mathrm{C}$. A working stock of the IS solution $(20 \mathrm{ng} / \mathrm{mL})$ was prepared in methanol and stored at $-20^{\circ} \mathrm{C}$.

\subsection{Instrument and Analytical Conditions}

Chromatography was performed on an Acquity ${ }^{\mathrm{TM}}$ UPLC system (Waters Corp, Milford, MA, USA) with an autosampler temperature at $10{ }^{\circ} \mathrm{C}$. Waters Acquity UPLC ${ }^{\circledR}$ HSS C18 column $(3.0 \times 50 \mathrm{~mm}, 1.8 \mu \mathrm{m}$ particle size $)$ was used for chromatographic separation with linear gradient elution consisting of (A) $90 \%$ acetonitrile and (B) $10 \% 0.2 \%$ formic acid in Milli-Q water as mobile phases. The flow rate was set at $0.30 \mathrm{~mL} / \mathrm{min}$, and the injection volume was $2 \mu \mathrm{L}$.

An Acquity Tandem Quadrupole Mass Detector (Xevo TQ-S; Waters Corp, Milford, MA, USA) in positive electrospray ionization mode was used for mass spectrometric detection. For collision-induced dissociation, argon was used as collision gas. The cone voltage and collision energy were set at $60 \mathrm{~V}$ and $34 \mathrm{~V}$ for $4 \mathbf{k}$ and $46 \mathrm{~V}$ and $26 \mathrm{~V}$ for the IS, respectively. Quantification was performed using the monitoring of multiple reaction 
monitoring (MRM) of following transitions: $m / z$ 409.0/220.9 for $4 \mathbf{k}$ and $m / z$ 180.0/92.7 for IS. Retention times of $4 \mathbf{k}$ and IS were 2.85 and $1.81 \mathrm{~min}$, respectively.

\subsection{Sample Preparation}

A simple protein precipitation method was followed for extraction of $4 \mathbf{k}$ from mice plasma. To an aliquot of $50 \mu \mathrm{L}$ of plasma or tissue (brain, liver, or kidney) samples, IS solution $(5 \mu \mathrm{L}$ of $20 \mathrm{ng} / \mathrm{mL}$ ) was added and mixed for $15 \mathrm{~s}$ on a cyclomixer (Thermo Scientific, Indianapolis, IN, USA). After precipitation with $200 \mu \mathrm{L}$ of acetonitrile, the mixture was vortexed for $2 \mathrm{~min}$, followed by centrifugation for $10 \mathrm{~min}$ at 14,000 rpm on an accuSpin Micro 17R (Fisher Scientific, Suwanee, GA, USA) at $5{ }^{\circ} \mathrm{C}$. An aliquot of $\sim 150 \mu \mathrm{L}$ of clear supernatant was transferred into vials and $2 \mu \mathrm{L}$ was injected onto LC-MS/MS system for analysis.

\subsection{In Vivo Studies in CD-1 Mice}

All work involving animal subjects was pre-approved by the University of Mississippi Institutional Animal Care and Use Committee and was conducted in accordance with ethical guidelines defined by the National Institutes of Health (NIH Publication No. 85-23).

\subsection{Subjects}

Male CD-1 mice ( $\mathrm{N}=48)$ were obtained from Envigo (St. Louis, MO, USA) and maintained on a 12:12 h reversed dark/light cycle (lights off at 07:00 h) with ad libitum access to food and water. Following a $\sim 4 \mathrm{~h}$ fast, animals were randomly assigned to one of two groups. Group I and II animals ( $\mathrm{n}=24$ /group, weight range $25-30 \mathrm{~g}$ ) received compound $4 \mathrm{k}(5 \mathrm{mg} / \mathrm{kg}$ ) orally (in the form of a solution, prepared using $10 \%$ absolute alcohol, $10 \%$ cremophor, and $80 \%$ Milli-Q water) or intravenously (i.v., using solution formulation comprising 10\% absolute alcohol, 10\% cremophor, and 80\% normal saline), respectively. At $0.5,1,2,4,8$, and $24 \mathrm{~h}$ post administration, animals were euthanized, blood was collected in $\mathrm{K}_{2}$. EDTA-containing polypropylene tubes and, tissues were collected (brain, liver, and kidney). Plasma was harvested by centrifuging the blood using Eppendorf 5430R Centrifuge (Germany) at $5000 \mathrm{rpm}$ for $5 \mathrm{~min}$ and stored frozen at $-80^{\circ} \mathrm{C}$ until analysis.

\subsection{Tissue Preparation}

Brain, liver, and kidney tissues were homogenized in separate $15 \mathrm{~mL}$ round-bottom screw-capped vials in phosphate buffered saline (5 volumes of each tissue mass) with a homogenizer (Polytron ${ }^{\circledR}$ ) and stored at $-80^{\circ} \mathrm{C}$ until analysis. Plasma or tissue homogenates $(50 \mu \mathrm{L})$ were spiked with IS and processed as mentioned in sample preparation section.

\subsection{Pharmacokinetic Assessments}

Plasma and tissue concentration and time data of compound $4 \mathbf{k}$ were analyzed by a non-compartmental method using WinNonlin Version 5.3 (Pharsight Corporation, Mountain View, CA, USA).

\section{Results and Discussion}

\subsection{Chemistry}

To gain a better understanding of the structure-activity relationships of 4 , we extended the range of PBD-11-hydrazinyl derivatives $(\mathbf{4} \mathbf{a}-\mathbf{4 q})$ by alkenylation of the primary amine group of the cycloamidine 3 via the formation of a Schiff's base with various carboxaldehydes [13]. Synthetic approaches were initiated based on the stepwise synthesis of dilactam $\mathbf{1}$ by the cyclocondensation of an equimolar mixture of L-proline and readily available basic structure of PBD natural product, isatoic anhydride (or substituted compound) in DMF at $155^{\circ} \mathrm{C}$. Thionation of compound 1 with 0.5 eq. of 2,4-bis-(4-methoxyphenyl)-1,3dithia-2,4-diphosphetane-2,4-disulfide (Lawesson's reagent) in THF at room temperature produced thiolactam 2 . The subsequent treatment of 2 with $98 \%$ hydrazine monohydrate in 
ethanol at room temperature generated cycloamindine 3 . The title compound 3 was further subjected to condensation with several aldehydes in anhydrous $\mathrm{MeOH}$ and molecular sieves ( $3 \AA$ ) at room temperature to afford a cluster of highly conjugated Schiff's base 4 in high yield. This procedure involves the initial formation of the intermediate carbinol amine, which dehydrates to form an imine. The reaction time was varied from a few minutes to a couple of hours and gave the desired products in good yields. The alkenylation of 3 with aldehydes was best performed under optimized conditions in which the parent molecule was exposed to five equivalents of aldehyde in methanol for varied reaction times at room temperature. Upon further crystallization of crude product from EtOAc/hexanes, pure $(S, E)-11-[2-($ arylmethylene)hydrazono]-PBD analogs $(\mathbf{4 a - 4 q})$ were formed as crystalline solids. The chemical structures are shown in Figure 2. These compounds are structurally unique from any reported small-molecule CB ligands, confirming the chemotype novelty of all the generated compounds.

\subsection{Biological Evaluation of the Synthesized Compounds}

\subsubsection{Cannabinoid Receptors Displacement Assay}

The CB1/CB2 receptor binding activities of analogs $\mathbf{4 a}-\mathbf{4 q}$ were assessed via radioactivitybased competitive binding assays using $\left[{ }^{3} \mathrm{H}\right] \mathrm{CP} 55,940$, an extensively studied radioligand that is frequently used for cannabinoid assays [14-16]. The highly potent and nonselective CB agonist CP55,940 binds to the same orthosteric active site where known CB agonists are known to bind. In preliminary screening, all 17 synthetic PBD compounds (4a-4q) were subjected to in vitro CB1 and CB2 binding assays at a single concentration of $10 \mu \mathrm{M}$. The observed percentage displacement (\%) of the radioligand at the CB receptors of these analogs are summarized in Table 1 . From the 17 compounds evaluated in the competitive radioligand assay, compounds $4 \mathbf{h}, \mathbf{4 j}-\mathbf{4 l}$, and $\mathbf{4 q}$ showed significant displacement (more than $60 \%$ ). Among them, two structurally distinct PBD analogs (4k and $4 \mathbf{q}$ ) displayed the greatest $\left[{ }^{3} \mathrm{H}\right] \mathrm{CP}-55,940$ displacement and selectivity for CB2 over CB1. Therefore, the most promising ligands (4k and $\mathbf{4 q}$ ) were selected for assessment in full competition curves against CB1 and CB2. The binding assays revealed selective binding affinity of $4 \mathbf{k}$ and $4 \mathbf{q}$ with $K_{\mathrm{i}}$ values of 146 and $137 \mathrm{nM}$, respectively, toward CB2 receptors (Table 2).

Table 1. Displacement of $\left[{ }^{3} \mathrm{H}\right] \mathrm{CP}-55,940$ from CB1 and CB2 receptors by $10 \mu \mathrm{M}$ concentration of compounds $\mathbf{4 a - 4 q}$.

\begin{tabular}{cccccc}
\hline Compound & CB1(\%) & CB2(\%) & Compound & CB1(\%) & CB2(\%) \\
\hline $\mathbf{4 a}$ & -3.09 & -12.50 & $\mathbf{4 j}$ & 66.81 & 65.24 \\
$\mathbf{4 b}$ & -13.99 & -14.48 & $\mathbf{4 k}$ & 41.26 & 84.60 \\
$\mathbf{4 c}$ & 0.25 & 3.06 & $\mathbf{4 1}$ & 77.17 & 51.73 \\
$\mathbf{4 d}$ & -27.99 & -33.65 & $\mathbf{4} \mathbf{m}$ & 5.22 & 50.05 \\
$\mathbf{4} \mathbf{e}$ & 21.88 & 19.86 & $\mathbf{4 n}$ & 44.14 & 43.34 \\
$\mathbf{4 f}$ & 1.75 & 3.72 & $\mathbf{4 o}$ & 5.09 & 1.01 \\
$\mathbf{4 g}$ & 24.19 & 29.27 & $\mathbf{4 p}$ & -27.20 & -29.47 \\
$\mathbf{4} \mathbf{h}$ & 36.88 & 61.05 & $\mathbf{4 q}$ & 55.27 & 95.58 \\
$\mathbf{4 i}$ & 49.82 & 36.39 & & & \\
\hline
\end{tabular}




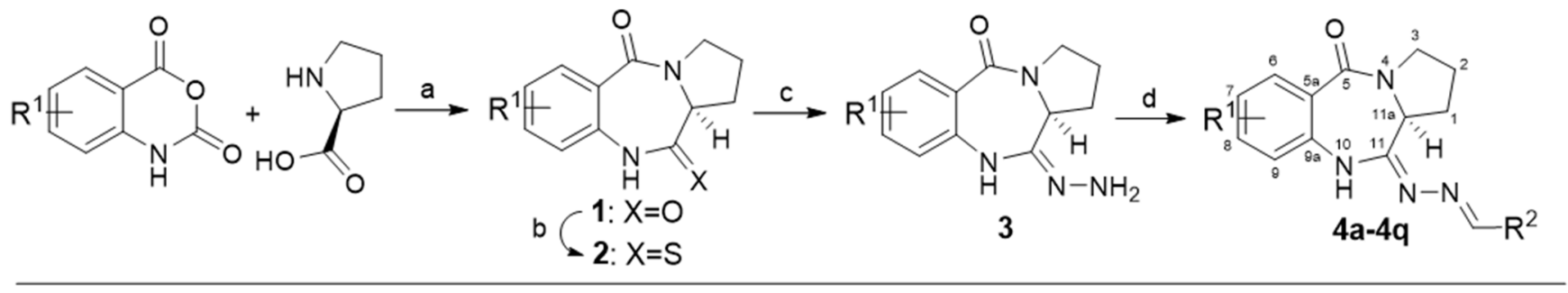

\begin{tabular}{|c|c|c|c|}
\hline Compound & $\mathrm{R}^{1}$ & $\mathbf{R}^{2}$ & Yield (\%) \\
\hline$(\mathrm{S}, \mathrm{E})-11$-[pyrrol-2-yl)methylene)hydrazono]-PBD (4a) & $\mathrm{H}$ & & 62 \\
\hline $\begin{array}{l}\text { (S,E)-11-[(1-methyl-1H-imidazol-2- } \\
\text { yl)methylene)hydrazono]-PBD (4b) }\end{array}$ & H & & 65 \\
\hline$(\mathrm{S}, \mathrm{E})-11-[($ thiazol-5-ylmethylene)hydrazono]-PBD (4c) & H & & 71 \\
\hline $\begin{array}{l}(\mathrm{S}, \mathrm{E})-11-[(2-\text { methylthiazol-5-yl)methylene)hydrazono]- } \\
\text { PBD (4d) }\end{array}$ & $\mathrm{H}$ & & 72 \\
\hline $\begin{array}{l}\text { (S,E)-11-[(4-methylthiazol-5-yl)methylene)hydrazono]- } \\
\text { PBD (4e) }\end{array}$ & $\mathrm{H}$ & & 61 \\
\hline $\begin{array}{l}\text { (S,E)-11-[(2-aminothiazol-5-yl)methylene)hydrazono]- } \\
\text { PBD (4f) }\end{array}$ & H & & 68 \\
\hline $\begin{array}{l}\text { (S,E)-11-[(2,4-dichlorothiazol-5-yl)methylene)hydrazono]- } \\
\text { PBD (4g) }\end{array}$ & H & & 69 \\
\hline $\begin{array}{l}\text { (S,E)-11-[2-(benzo[b]thiophenylmethylene)hydrazono]- } \\
\text { PBD (4h) }\end{array}$ & $\mathrm{H}$ & & 75 \\
\hline $\begin{array}{l}\text { (S,E)-11-[(benzo[b]thiophen-2- } \\
\text { ylmethylene))hydrazono]-7-bromo-PBD (4i) }\end{array}$ & 7-bromo & & 70 \\
\hline $\begin{array}{l}(\mathrm{S}, \mathrm{E})-11-[(\text { benzo[b]thiophen-3- } \\
\text { ylmethylene))hydrazono]-7-bromo-PBD (4j) }\end{array}$ & 7-bromo & & 72 \\
\hline $\begin{array}{l}\text { (S,E)-11-[(benzo[b]thiophen-2- } \\
\text { ylmethylene)hydrazono]-8-chloro-PBD }(\mathbf{4 k})\end{array}$ & 8-chloro & & 75 \\
\hline $\begin{array}{l}\text { (S,E)-11-[(3-methylbenzo[b]thiophen-2-yl)methylene)hydrazono]- } \\
\text { PBD (4I) }\end{array}$ & $\mathrm{H}$ & & 65 \\
\hline $\begin{array}{l}\text { (S,E)-11-[(3-chlorobenzo[b]thiophen-2-yl)methylene)hydrazono]- } \\
\text { PBD }(4 \mathrm{~m})\end{array}$ & $\mathrm{H}$ & & 60 \\
\hline $\begin{array}{l}\text { (S,E)-11-[(benzo[d]thiazol-2-ylmethylene)hydrazono]- } \\
\text { PBD (4n) }\end{array}$ & $\mathrm{H}$ & & 68 \\
\hline $\begin{array}{l}(\mathrm{S}, \mathrm{E})-11-[(1-\text { methyl-1H-indol-3- } \\
\text { yl)methylene }) \text { hydrazono]-PBD (4o) }\end{array}$ & $\mathrm{H}$ & & 69 \\
\hline $\begin{array}{l}\text { (S,E)-11-[(5-methoxy-1H-indol-3-yl)methylene }) \text { hydrazono]-PBD } \\
(\mathbf{4 p})\end{array}$ & $\mathrm{H}$ & & 68 \\
\hline $\begin{array}{l}\text { (S,E)-11-[(adamantan-1-yl)methylene)hydrazono]- } \\
\text { PBD (4q) }\end{array}$ & $\mathrm{H}$ & & 68 \\
\hline
\end{tabular}

Figure 2. Reagents and conditions: (a) DMF, $155{ }^{\circ} \mathrm{C}, 5 \mathrm{~h}, 82.0 \%$; (b) Lawesson's reagent, $\mathrm{THF}, \mathrm{rt}$, 15 h, 87.0\%; (c) $\mathrm{N}_{2} \mathrm{H}_{4} \cdot \mathrm{H}_{2} \mathrm{O}$ (98\%), EtOH(abs.), rt, 15 h, 99.0\%; (d) Aldehydes, $\mathrm{MeOH}$ (anhy.), rt, 15 h, $60-75.0 \%$. 
Table 2. Cannabinoid receptor binding assays of the lead compounds against CB2.

Compound

\subsubsection{In Vivo PK Studies of the Lead Analog 4k}

Drug discovery research programs routinely execute mouse PK studies to characterize the PK properties of compounds as a filtering tool to advance drug candidates through the pipeline as well as to support efficacy and toxicology studies. Stability studies on two selected analogs has revealed a better tolerance of $4 \mathbf{k}$ under physiological $\mathrm{pH}$ when compared to $\mathbf{4 q}$ compound (not reported). Consequently, $4 \mathbf{k}$ was selected for pharmacokinetic studies in CD1 male mice at $5 \mathrm{mg} / \mathrm{kg}$ body weight (Table 3, Figure 3).

Table 3. Pharmacokinetic parameters of compound $4 \mathbf{k}$ in CD1 mice following intravenous and oral dosing of $4 \mathbf{k}$ in mice at $5 \mathrm{mg} / \mathrm{kg}$.

\begin{tabular}{|c|c|c|c|c|c|c|c|c|c|}
\hline \multicolumn{2}{|c|}{ PK Parameters } & \multicolumn{4}{|c|}{ Intravenous } & \multicolumn{4}{|c|}{ Oral } \\
\hline & & Plasma & Brain & Kidney & Liver & Plasma & Brain & Kidney & Liver \\
\hline$t_{1 / 2}$ & $\mathrm{~h}$ & 6.91 & 6.54 & 10.2 & 9.26 & 16.4 & 22.9 & 16.7 & 13.0 \\
\hline $\mathrm{C}_{\max }$ or $\mathrm{C}_{0}$ & $\mathrm{ng} / \mathrm{mL}$ or $\mathrm{ng} / \mathrm{g}$ & 4345 & 2260 & 4725 & 13497 & 198 & 602 & 795 & 1891 \\
\hline $\mathrm{T}_{\max }$ & $\mathrm{h}$ & - & - & - & - & 0.88 & 1.25 & 0.75 & 0.75 \\
\hline $\mathrm{AUC}_{0-\infty}$ & $\begin{array}{c}\mathrm{ng} \times \mathrm{h} / \mathrm{mL} \text { or } \\
\mathrm{ng} \times \mathrm{h} / \mathrm{g}\end{array}$ & 4015 & 5685 & 13272 & 19991 & 739 & 3069 & 5223 & 6423 \\
\hline $\mathrm{CL}$ & $\mathrm{mL} / \mathrm{min} / \mathrm{kg}$ & 23.5 & 15.7 & 6.38 & 4.28 & - & - & - & - \\
\hline $\mathrm{V}_{\mathrm{d}}$ & $\mathrm{L} / \mathrm{kg}$ & 14.3 & - & - & - & - & - & - & - \\
\hline $\mathrm{F}$ & $\%$ & - & - & - & - & 18 & 43 & 37 & 32 \\
\hline
\end{tabular}



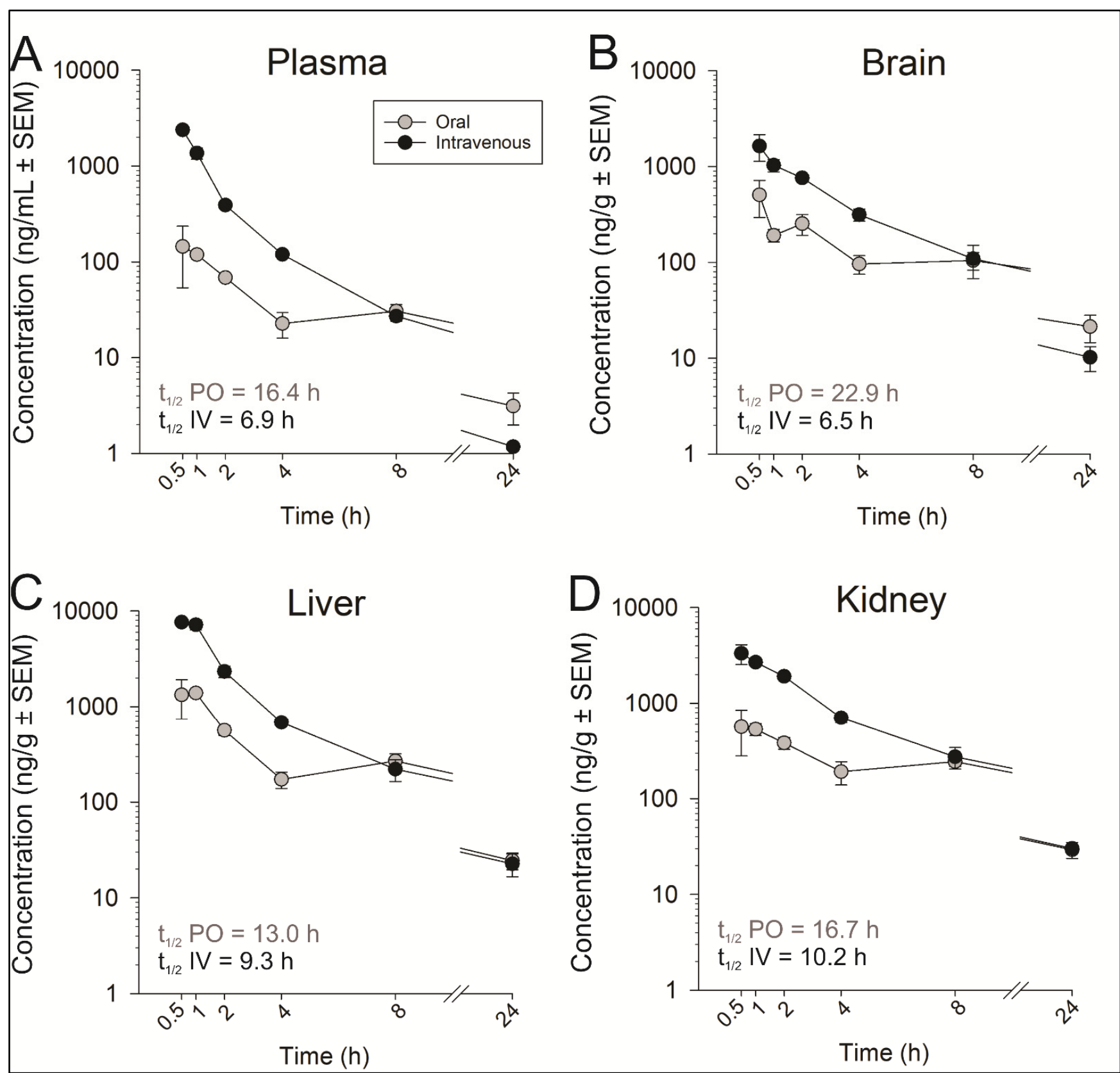

Figure 3. Pharmacokinetic profiles of compound $4 \mathbf{k}$ in plasma (A), brain (B), liver (C), and kidney (D) of CD1 mice following intravenous and oral dosing of $4 \mathbf{k}$ to mice at $5 \mathrm{mg} / \mathrm{kg}$.

In plasma, the concentrations of compound $4 \mathbf{k}$ decreased in a mono-exponential manner after $5.0 \mathrm{mg} / \mathrm{kg}$ intravenous administration. The mean clearance (CL) was found to be $23.5 \pm 5.35 \mathrm{~mL} / \mathrm{min} / \mathrm{kg}$, which is $26 \%$ of hepatic blood flow in mice. Compound $4 \mathrm{k}$ had a high volume of distribution of $14.3 \pm 3.11 \mathrm{~L} / \mathrm{kg}$. The terminal half-life $\left(\mathrm{t}_{1 / 2}\right)$ was found to be $6.91 \pm 0.15 \mathrm{~h}$. The post oral administration, maximum plasma concentrations $\left(\mathrm{C}_{\max }: 198 \pm 11.51 \mathrm{ng} / \mathrm{mL}\right)$ were attained at $0.88 \pm 0.13 \mathrm{~h}\left(\mathrm{~T}_{\max }\right)$, indicating rapid absorption from the gastrointestinal tract. The apparent half-life $(16.4 \pm 1.66 \mathrm{~h})$ determined after oral administration was longer than that after intravenous administration $(6.91 \mathrm{~h})$, which may indicate multiple sites of absorption. The $\mathrm{AUC}_{0-\infty}$ attained post oral dose was $739 \pm 6.75 \mathrm{ng} \times \mathrm{h} / \mathrm{mL}$. The oral bioavailability in mice at $5 \mathrm{mg} / \mathrm{kg}$ was $18 \pm 2.43 \%$ (Table 3). 
In the brain, the concentrations of compound $4 \mathbf{k}$ decreased mono-exponentially after $5.0 \mathrm{mg} / \mathrm{kg}$ intravenous administration. The mean brain clearance (CL) was found to be $15.7 \pm 2.31 \mathrm{~mL} / \mathrm{min} / \mathrm{kg}$. Compound $4 \mathrm{k}$ had a high volume of distribution of $8.82 \pm 2.43 \mathrm{~L} / \mathrm{kg}$ in the brain. The terminal half-life $\left(\mathrm{t}_{1 / 2}\right)$ was found to be $6.54 \pm 1.38 \mathrm{~h}$. Post oral administration, the maximum brain concentrations $\left(C_{\max }: 602 \pm 44.75 \mathrm{ng} / \mathrm{g}\right)$ were attained at $1.25 \pm 0.44 \mathrm{~h}\left(\mathrm{~T}_{\max }\right)$. The apparent half-life was found to be $22.9 \pm 3.73 \mathrm{~h}$. The $\mathrm{AUC}_{0-\infty}$ attained post oral dose was $3069 \pm 64 \mathrm{ng} \times \mathrm{h} / \mathrm{g}$. The apparent oral bioavailability to the brain, as compared to IV administration, was $42.5 \pm 5.75 \%$ (Table 1 ). The mean brain to plasma ratios after intravenous and oral administration were 3.67 and 4.61, respectively, which indicates that the compound had very good brain penetration.

In the kidney, the concentrations of compound $4 \mathbf{k}$ decreased mono-exponentially after $5.0 \mathrm{mg} / \mathrm{kg}$ intravenous administration. The mean kidney clearance (CL) was found to be $6.38 \pm 0.49 \mathrm{~mL} / \mathrm{min} / \mathrm{kg}$. Compound $4 \mathbf{k}$ had a high volume of distribution of $5.63 \pm 0.37 \mathrm{~L} / \mathrm{kg}$ in the kidney. The terminal half-life $\left(\mathrm{t}_{1 / 2}\right)$ was found to be $10.2 \pm 0.49 \mathrm{~h}$. Post oral administration, the maximum kidney concentrations $\left(C_{\max }: 795 \pm 22.65 \mathrm{ng} / \mathrm{g}\right)$ were attained at $0.75 \pm 0.15 \mathrm{~h}\left(\mathrm{~T}_{\max }\right)$. The apparent half-life was found to be $16.7 \pm 1.61 \mathrm{~h}$. The $\mathrm{AUC}_{0-\infty}$ attained post oral dose was $5223 \pm 44.75 \mathrm{ng} \times \mathrm{h} / \mathrm{g}$. The apparent oral bioavailability in the kidney at $5 \mathrm{mg} / \mathrm{kg}$ was $37 \pm 3.78 \%$ (Table 1). The mean kidney to plasma ratios after intravenous and oral administration were 14.5 and 7.25 , respectively.

In the liver, the mean liver clearance (CL) was found to be $4.28 \pm 0.38 \mathrm{~mL} / \mathrm{min} / \mathrm{kg}$. Compound $4 \mathbf{k}$ had a high volume of distribution of $3.38 \pm 0.18 \mathrm{~L} / \mathrm{kg}$ in liver. The terminal half-life $\left(t_{1 / 2}\right)$ was found to be $9.26 \pm 0.67 \mathrm{~h}$. Post oral administration, the maximum liver concentrations $\left(\mathrm{C}_{\max }: 1891 \pm 282 \mathrm{ng} / \mathrm{g}\right)$ were attained at $0.75 \pm 0.15 \mathrm{~h}\left(\mathrm{~T}_{\max }\right)$. The apparent half-life was found to be $13.0 \pm 0.97 \mathrm{~h}$. The $\mathrm{AUC}_{0-\infty}$ attained post oral dose was $6423 \pm 571 \mathrm{ng} \times \mathrm{h} / \mathrm{g}$. The apparent oral bioavailability in the liver at $5 \mathrm{mg} / \mathrm{kg}$ was $32 \pm 2.86 \%$ (Table 1 ). The mean liver to plasma ratios after intravenous and oral administration were 11.5 and 9.89 , respectively.

\section{Conclusions}

Through a structure-based rational drug design, we synthesized a subset of $17(S, E)$ 11-[2-(arylmethylene)hydrazono]-PBD derivatives using a multi-step synthesis approach to establish clusters of highly potent and selective CB2 ligands. Most of the designed analogs exhibited a high percentage displacement (\%) of the CB1 and CB2 receptor. Most importantly, the most promising compounds, $\mathbf{4 k}$ and $\mathbf{4 q}$, displayed sub-micromolar efficacy ( $K_{\mathrm{i}}$ of 146 and $137 \mathrm{nM}$ ) when tested for in a binding affinity assay.

Our previously reported studies have shown suitable drug-like properties of this class of ligands via the computational calculation of ADMET and physicochemical properties. The reported data validated that almost all the designed analogs possess Abs_risk, CYP_Risk, TOX_Risk, and ADMET_Risk within the satisfactory defined limits [13]. The current pharmacokinetics assessments further support the favorable drug-like behavior of this class in that the oral bioavailability is reasonable and tissue uptake, especially brain penetration, is high, with a prolonged half-life. This suggests the feasibility of central nervous system activity with simple dose regimens. The structure-activity relationships (SAR) suggested that attachment of S-heterocyclic aldehydes (e.g., benzothiophene) to the hydrazine portion, in addition to an 8-chloro-substituted PBD, improves the selectivity of CB2 over CB1. Moreover, the adamantyl pharmacophore establishes extensive hydrophobic interactions with three phenylalanine residues and one histidine residue within the active site of the CB2 receptor [17]. Most notably, these bioactive compounds represent structurally new chemotypes in the area of cannabinoid research and could be considered for further structural optimization as selective CB2 ligands.

Supplementary Materials: The following supporting information can be downloaded online. Spectroscopic data for PBD analogs (4a-4q) and typical MRM chromatograms of $4 \mathbf{k}$ in plasma and tissues. 
Author Contributions: Conceptualization, J.J.P., A.G.S. and M.A.I.; methodology, M.O., S.P.S., J.J.P., J.M.R., N.A., A.G.S. and M.A.I.; software, M.O., S.P.S. and J.J.P.; validation, S.P.S., J.J.P., L.W. and M.A.I.; formal analysis, M.O., S.P.S., J.J.P. and M.A.I.; investigation, M.O., S.P.S., J.J.P., L.W., S.A.R., A.G.S. and M.A.I.; data curation, S.P.S., J.J.P. and M.A.I.; writing-original draft preparation, M.O., S.P.S., A.G.S. and M.A.I.; writing-review and editing, M.O., S.P.S., J.J.P., L.W., S.A.R., A.G.S. and M.A.I.; supervision, L.W., S.A.R. and M.A.I.; funding acquisition, M.A.I. All authors have read and agreed to the published version of the manuscript.

Funding: This work is supported by the National Institute of General Medical Science of the National Institute of Health under Award Number P30GM122733. The content is solely the responsibility of the authors and does not necessarily represent the official views of the National Institutes of Health.

Institutional Review Board Statement: Not applicable.

Informed Consent Statement: Not applicable.

Data Availability Statement: Spectroscopic data for PBD analogs (4a-4q) and MRM chromatograms of $4 \mathbf{k}$ in plasma and tissues are available in the supplementary materials.

Acknowledgments: The authors are thankful to the Neuropharmacology CORE (CORE-NPN) and the Chemistry and Drug Metabolism Pharmacokinetics (DMPK) CORE, School of Pharmacy, University of Mississippi for biological testing and PK analysis. The authors acknowledge the Department of Chemistry and The School of Graduate Studies at ETSU.

Conflicts of Interest: The authors declare no conflict of interest.

Sample Availability: Samples of the compounds are available from the authors.

\author{
Abbreviations \\ CB2 Cannabinoid Receptor Subtype 2 \\ PBD Pyrrolo[2,1-c][1,4]benzodiazepines \\ PK Pharmacokinetics
}

\title{
References
}

1. Gao, H.M.; Hong, J.S. Why neurodegenerative diseases are progressive: Uncontrolled inflammation drives disease progression. Trends Immunol. 2008, 29, 357-365. [CrossRef] [PubMed]

2. Brettschneider, J.; Tredici, K.D.; Lee, V.M.; Trojanowski, J.Q. Spreading of pathology in neurodegenerative diseases: A focus on human studies. Nat. Rev. Neurosci. 2015, 16, 109-120. [CrossRef] [PubMed]

3. McPartland, J.M. Phylogenomic and chemotaxonomic analysis of the endocannabinoid system. Brain Res. Rev. 2004, 45, 18-29. [CrossRef] [PubMed]

4. Beltramo, M.; Bernardini, N.; Bertorelli, R.; Campanella, M.; Nicolussi, E.; Fredduzzi, S.; Reggiani, A. CB2 receptor-mediated antihyperalgesia: Possible direct involvement of neural mechanisms. Eur. J. Neurosci. 2006, 23, 1530-1538. [CrossRef] [PubMed]

5. Mukhopadhyay, S.; Das, S.; Williams, E.A.; Moore, D.; Jones, J.D.; Zahm, D.S.; Ndengele, M.M.; Lechner, A.J.; Howlett, A.C. Lypopolysaccharide and cyclic AMP regulation of CB2 cannabinoid receptor levels in rat brain and mouse RAW 264.7 macrophages. J. Neuroimmunol. 2006, 181, 82-92. [CrossRef] [PubMed]

6. Klein, T.W. Cannabinoid-based drugs as anti-inflammatory therapeutics. Nat. Rev. Immunol. 2005, 5, 400-411. [CrossRef] [PubMed]

7. Nakagawa, Y.; Chiba, K. Role of microglial $\mathrm{m} 1 / \mathrm{m} 2$ polarization in relapse and remission of psychiatric disorders and diseases. Pharmaceuticals 2014, 7, 1028-1048. [CrossRef] [PubMed]

8. Hebert, L.E.; Weuve, J.; Scherr, P.A.; Evans, D.A. Alzheimer disease in the United States (2010-2050) estimated using the 2010 census. Neurology 2013, 80, 1778-1783. [CrossRef] [PubMed]

9. Wu, J.; Bie, B.; Yang, H.; Xu, J.J.; Brown, D.L.; Naguib, M. Activation of the CB2 receptor system reverses amyloid-induced memory deficiency. Neurobiol. Aging 2013, 34, 791-804. [CrossRef] [PubMed]

10. Schmidt, A.; Shilabin, A.G.; Nieger, M. Syntheses and tautomerization of amino-substituted and pyrimidine-annulated pyrrolobenzodiazepines. Heterocycles 2005, 65, 625-632. [CrossRef]

11. Schmidt, A.; Shilabin, A.G.; Namyslo, J.C.; Nieger, M.; Hemmen, S. Pyrimidine-annulated Pyrrolobenzodiazepines. A New Ring System Related to Aspergillus Alkaloids. Eur. J. Org. Chem. 2005, 1781-1789. [CrossRef]

12. Cipolla, L.; Araújo, A.C.; Airoldi, C.; Bini, D. Pyrrolo[2,1-c][1,4]benzodiazepine as a scaffold for the design and synthesis of anti-tumour drugs. Anticancer Agents Med. Chem. 2009, 9, 1-31. [CrossRef] [PubMed] 
13. Mingle, D.; Ospanov, M.; Radwan, M.O.; Ashpole, N.; Otsuka, M.; Ross, S.A.; Walker, L.; Shilabin, A.G.; Ibrahim, M.A. First In Class (S,E)-11-[2-(Arylmethylene)Hydrazono]-PBD Analogs As Selective CB2 Modulators Targeting Neurodegenerative Disorders. Med. Chem. Res. 2020, 1-11. [CrossRef] [PubMed]

14. Dean, B.; Sundram, S.; Bradbury, R.; Scarr, E.; Copolov, D. Studies on $\left[{ }^{3} \mathrm{H}\right] \mathrm{CP}-55940$ binding in the human central nervous system: Regional specific changes in density of cannabinoid-1 receptors associated with schizophrenia and cannabis use. Neuroscience 2001, 103, 9-15. [CrossRef]

15. Stempel, A.V.; Stumpf, A.; Zhang, H.Y.; Ozdogan, T.; Pannasch, U.; Theis, A.K.; Otte, D.M.; Wojtalla, A.; Racz, I.; Ponomarenko, A.; et al. Cannabinoid type 2 receptors mediate a cell type-specific plasticity in the hippocampus. Neuron 2016, 90, 795-809. [CrossRef] [PubMed]

16. Jayant, S.; Sharma, B.M.; Bansal, R.; Sharma, B. Pharmacological benefits of selective modulation of cannabinoid receptor type 2 (CB2) in experimental Alzheimer's disease. Pharmacol. Biochem. Behav. 2016, 140, 39-50. [CrossRef] [PubMed]

17. Li, X.; Hua, T.; Vemuri, K.; Ho, J.H.; Wu, Y.; Wu, L.; Popov, P.; Benchama, O.; Zvonok, N.; Qu, L.; et al. Crystal structure of the human cannabinoid receptor CB2. Cell 2019, 176, 459-467. [CrossRef] [PubMed] 